\title{
Developmental Hemodynamic Changes in Rat Embryos at 11 to 15 Days of Gestation: Normal Data of Blood Pressure and the Effect of Caffeine Compared to Data from Chick Embryo
}

\author{
MAKOTO NAKAZAWA, SACHIKO MIYAGAWA, TAKASHI OHNO, SHOUJI MIURA, AND \\ ATSUYOSHI TAKAO \\ Department of Pediatric Cardiology, The Heart Institute of Japan, Tokyo Women's Medical College, \\ Tokyo, Japan
}

\begin{abstract}
We attempted to measure arterial blood pressure of the rat embryo. The embryo was excised within the uterus and immersed in Hanks' solution at $37^{\circ} \mathrm{C}$. The uterus wall and yolk sac were opened to expose the umbilical vessels. The umbilical artery was punctured with a glass micro-pipette, and blood pressure was measured by using a servo-null micro-pressure system. The mean blood pressure was $0.27 \pm 0.05 \mathrm{~mm} \mathrm{Hg}$ in the embryo at the 11th day of gestation $(n=7), 0.48 \pm 0.03 \mathrm{~mm} \mathrm{Hg}$ in the 12-day embryos $(n=19), 1.3 \pm 0.08 \mathrm{~mm} \mathrm{Hg}$ in the 13day $(n=11)$, and $2.6 \pm 0.1 \mathrm{~mm} \mathrm{Hg}$ in the 15-day embryos $(n=10)$. Heart rate was $84 \pm 11$ in 11-day, $122 \pm 3$ in 12-day, $192 \pm 7$ in 13-day, and $198 \pm 5$ in 15-day embryos. These parameters were stable within $10 \mathrm{~min}$ after the excision. A comparison of the data with those of the chick embryo of comparable developmental stages revealed that the blood pressure was lower in 11- and 12-day rat embryos than in the chick embryo of Hamburger-Hamilton stages 18 and 21, but this was reversed in the later stages. In the stage 21 chick embryo, intravenous administration of caffeine $(60 \pm 9 \mathrm{mg} / \mathrm{kg}$ embryo weight) induced an increase in blood pressure by $11 \pm 3 \%(n=8)$, but did not result in a significant increase in dorsal aortic blood flow (6 \pm $6 \%, n=9)$ or in heart rate. In contrast, caffeine $(62 \pm 3$ $\mathrm{mg} / \mathrm{kg})$ increased the heart rate by $8 \pm 2 \%(n=10)$ without changing the blood pressure in the rat embryo of day 12 . The velocity of blood flow in the truncus was measured by a pulsed Doppler flowmeter. Caffeine injection increased the mean velocity by $21 \pm 8 \%(n=8)$. Herein we indicate that measurement of blood pressure in the rat embryo is feasible, but with some limitations, and that there may be qualitative hemodynamic differences between the rat and chick embryos. (Pediatr Res 23: 200-205, 1988)
\end{abstract}

While searching for the basic mechanism of normal and abnormal morphogenesis of the cardiovascular system, several investigators have been trying to correlate the change of hemodynamics or rheology of the cardiovascular system to morphogenesis $(1-5)$. These studies have revealed that several teratogenic factors alter the hemodynamics (6-12). However, it is known that caffeine induces aortic aneurysm in the chick (13), whereas

Received June 11, 1987; accepted October 8, 1987.

Correspondence Makoto Nakazawa, M.D., Department of Pediatric Cardiology, The Heart Institute of Japan, Tokyo Women's Medical College, 8-1 Kawada-cho, Shinjuku-ku, Tokyo, 162 Japan. in the rat it induces ventricular septal defect $(14,15)$. This indicates that there is a species difference in the teratogenecity of a single intervention. Previous hemodynamic studies, however, have only used the chick embryo, and very few have been done in the mammalian embryo. The ultimate goal of investigation in this field is an understanding of human cardiac morphogenesis. Experiments using any mammalian species may be better for this purpose than those using other species. Therefore, we attempted to measure the blood pressure and blood flow parameters in the rat embryo, and looked at whether there was any difference in the hemodynamic effect of caffeine in the chick and rat embryo.

\section{MATERIALS AND METHODS}

The Wistar rat was used and pregnancy was confirmed by vaginal smear on the day after overnight mating, when the gestational day was counted as day zero.

The pregnant rat was anesthetized by intraperitoneal injection of $\alpha$-chloralose, $80 \mathrm{mg} / \mathrm{kg}$ of body weight, at $11,12,13$, and 15 days of gestation. The uterus was exposed through abdominal incision and then a part was excised from the ovarian end containing one embryo. The vessels to the uterus were ligated each time a part was excised.

The uterus containing the embryo was immersed into Hanks' solution at $37^{\circ} \mathrm{C}$, and the temperature was monitored by a needle thermoprobe kept in the bath. Within the bath, the uterus wall was opened, and the wall was pinned down on a rubber sheet in a glass dish. A small window was made at the base of the yolk sac to expose the umbilical vessels. The umbilical artery was punctured using a glass micro-pipette, $5-10 \mu \mathrm{m}$ in tipdiameter, which was connected to a servo-null micropressure system (WP instrument, model 900) (Fig. 1). During this procedure, special care was taken to avoid bleeding. It took 4-6 min to complete cannulation from excision in the 12-, 13-, and 15day embryos, but it took an average of $7.5 \mathrm{~min}$ in the 11-day embryos.

The Hanks' solution used in the present study contained $8 \mathrm{~g}$ $\mathrm{NaCl}, 0.4 \mathrm{~g} \mathrm{KCl}, 0.09 \mathrm{~g} \mathrm{Na}_{2} \mathrm{HPO}_{4} 7 \mathrm{H}_{2} \mathrm{O}$ ), $0.06 \mathrm{~g} \mathrm{Na}_{2} \mathrm{PO}_{4} 2 \mathrm{H}_{2} \mathrm{O}$, $0.06 \mathrm{~g} \mathrm{KH}_{2} \mathrm{PO}_{4}, 0.35 \mathrm{~g} \mathrm{NaHCO}_{3}$, and $1 \mathrm{~g}$ glucose in 1 liter of distilled water.

A total of 12 pregnant rats was used and the data were successfully obtained in 50 embryos; seven embryos at 11 days of gestation from four litters, 19 embryos at 12 days from three litters, 12 embryos at 13 days from three litters, and 12 embryos at 15 days from two litters. We excluded the embryo unless cannulation was completed at the first trial or within $10 \mathrm{~min}$ after excision from the mother rat. 
We determined if the time from the beginning of laparotomy of the mother rat until removal of each embryo could affect the physical condition of the embryos. Taking heart rate as an indicator, we found that it was in a relatively narrow range for each litter when the embryos were excised one by one within approximately $2 \mathrm{~h}$ after the laparotomy (Fig. 2). One 15-day embryo showed an extremely low heart rate even though it was excised within $2 \mathrm{~h}$. This and the other two embryos that had an extremely low heart rate were excluded from the following discussion (indicated by an arrow in Fig. 2), because they were considered deteriorated. Body wet weight, heart weight, and CR length of each group are listed in Table 1.

In the separate groups of embryos, we observed the effects of intravenous injection of caffeine on heart rate, blood pressure, and blood flow parameters, and compared them to those in the

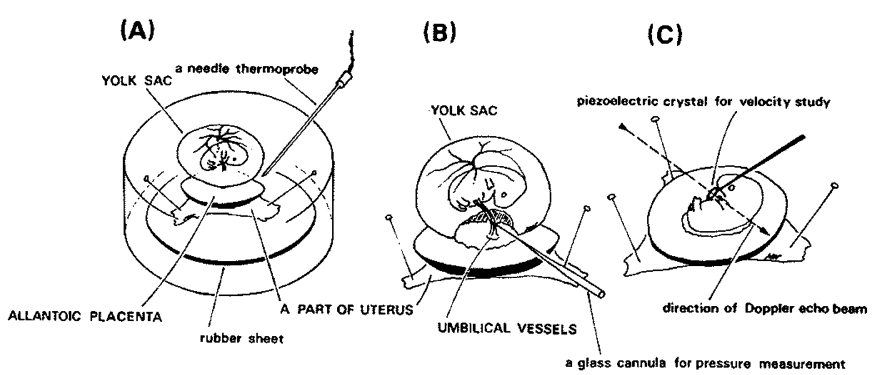

Fig. 1. Schematic illustration of the preparation. The embryo was excised within the uterus and immersed in Hanks' solution at $37^{\circ} \mathrm{C}$. The uterus wall was opened and a part was pinned down on a rubber sheet at the bottom of the dish $(A)$. The yolk sac was windowed to expose the umbilical vessels. The artery was cannulated with a glass micro-pipette connected to a servonull micropressure system $(B)$. When the flow velocity was measured, the whole embryo was exposed. Then, a micropiezo-electric probe was positioned as the echo beam passed through the axis of the truncus of the embryonic heart. The velocity was measured with a 20-MHz pulsed Doppler flowmeter $(C)$.

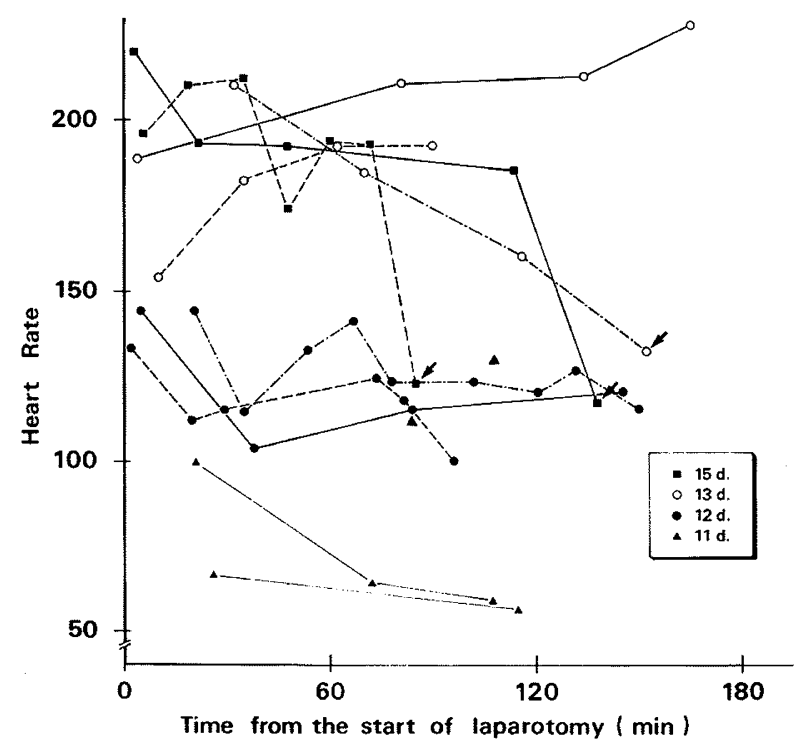

Fig. 2. Heart rate is plotted against the time in min from when the abdominal wall was first opened. Each point represents one embryo and each line indicates one litter. Embryos of gestational day 15 are expressed by closed squares, day 13 by open circles, day 12 by closed circles, and day 11 by closed triangles. Heart rate was relatively constant in embryos of the same litter excised within $2 \mathrm{~h}$ from the beginning of laparotomy. Two embryos of day 15 and one of day 13 (arrow) were excluded from the statistics because their extremely low heart rate was indicative of deterioration of their physical condition. chick embryo. Matsuoka et al. (15) reported that caffeine, given to a pregnant rat, induced an anomaly complex in the fetus including cardiovascular defects and that the most vulnerable day of gestation was 11 , followed by day 12 . Inasmuch as obtaining hemodynamic data in a day- 11 embryo was extremely difficult, we used day-12 embryos in this study. According to Sissman's table, the Hamburger-Hamilton (17) stage 21 chick embryo was very close to the day- 12 rat embryo in the aspect of morphological development. In the chick embryo, vitelline arterial blood pressure and blood flow at the dorsal aorta were measured, as described by Clark and $\mathrm{Hu}(18)$.

Inasmuch as cardiac output could not be obtained in the rat embryo (19), the blood flow velocity was measured at the truncus of the embryonic heart by using a $20-\mathrm{MHz}$ pulsed Doppler flowmeter (University of Iowa) with a micro-probe $1 \mathrm{~mm}$ in diameter (Fig. 3). The yolk sac was opened widely in Hanks' solution to expose the embryo's chest. In this stage of gestation, the heart and truncal region were clearly visible through the thoracic wall. The probe was then positioned as the Doppler beam passed through the center of the long axis of the truncus (Fig. 1). When positioning the probe, the largest signal of forward flow was searched as we adjusted the direction of the Doppler beam by a micro-manipulator. In some embryos, we had to readjust the position of the probe to obtain the maximum signal during the experiment. From the spatial relation of the cardiovascular system of this developmental stage, it seemed that no other flow gave any velocity faster than that in the conotruncal region in this Doppler beam direction. If the probe was directed too caudally, a reversed signal was recorded just before the forward flow. This reversed flow was confirmed to be atrial flow into the primitive ventricle from the following observation. We were able to separate the signal of ventricular output from that of atrial output by looking at the heart under a microscope while simultaneously listening to the Doppler sound when 2 to 1 atrioventricular block occurred late in the experiment (Fig. 4). When this reversed signal could not be completely avoided, forward flow was calculated by integrating the area underneath the positive signal.

In the embryo of this gestational age (day 12), the conotruncal swelling is already present (16). It may be argued that this swelling interferes with the flow profile that causes an error in the velocity data. Observations in the chick embryo have indicated that conotruncal flow is laminar but not turbulent in the stage of development of the conotruncal swelling $(3,6)$. In the rat embryo, it was observed under a microscope that the conotruncal region was fully patent during ventricular ejection and almost completely obliterated during ventricular diastole; this finding was

Table 1. Materials, wet wt, CR length, heart wt (mean $\pm S E M$ )

\begin{tabular}{lcccc}
\hline \multicolumn{1}{c}{ Gestational day } & 11 & 12 & 13 & 15 \\
\hline$n$ & 7 & 19 & 11 & 10 \\
CR length (mm) & $4.2 \pm 0.1$ & $7.0 \pm 0.09$ & $9.3 \pm 0.2$ & $13.4 \pm 0.11$ \\
Body wet wt (mg) & $8.0 \pm 0.6$ & $35.0 \pm 1.1$ & $87.1 \pm 4.8$ & $286 \pm 6$ \\
Heart wet wt (mg) & $0.16 \pm 0.04$ & $0.28 \pm 0.02$ & $0.62 \pm 0.04$ & $1.4 \pm 0.09$ \\
\hline
\end{tabular}

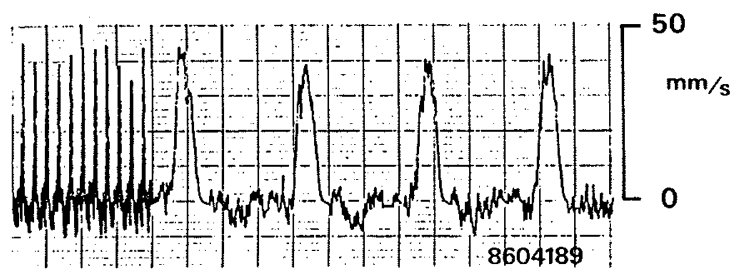

Fig. 3. The flow velocity pattern obtained at the truncus of the rat embryo heart of gestational day 12 . Note that there is only a minimum negative signal during diastole indicating that the conotruncal swelling has valve function. 
similarly observed when caffeine was injected. We therefore considered that the conotruncal swelling did not produce any large turbulence or had only a little effect on the flow profile if any. Thus, the change in cardiac output was estimated by the change in mean velocity assuming that the intervention did not cause any change in the diameter of the truncus.

Two micrograms of caffeine dissolved in distilled water were infused in a 100-nl solution over a 1 -min period by using a nanoliter pump (WP instrument). It was infused into the first or second order of the vitelline vein in the chick embryo and into the fetal side of the allantoic placenta in the rat embryo. The fetal placenta is composed of allantoic (umbilical) capillaries and well vascularized by gestational day 12 . Thus, this part of the placenta is in direct communication with the embryonic circulation via the umbilical artery and vein (20), and injection of the drug to this part has been considered equivalent to intravenous injection. The amount of caffeine given was $60 \pm 9 \mathrm{mg} / \mathrm{kg}$ of body weight in the chick embryo and $62 \pm 3 \mathrm{mg} / \mathrm{kg}$ in the rat embryo. In order to evaluate the effect of the volume infusion itself, $100 \mathrm{nl}$ of chick Ringer solution or Hanks' solution were infused for $1 \mathrm{~min}$ into the chick and rat embryo, respectively.

The data are expressed as mean \pm SE. When the effect of caffeine was tested, Student's paired $t$ test was used, and $p<0.05$ was considered significant.

\section{RESULTS}

Control study. Heart rate and blood pressure were constant until $10 \mathrm{~min}$ after excision in the 12-day group. However, the heart rate declined by 4 and $9 \%$ and the blood pressure declined by 0.2 and $0.3 \mathrm{~mm} \mathrm{Hg}$ in the 13- and 15-day embryos, respectively, although the change was not statistically significant. The heart rate of the 11-day embryo decreased with time, but the blood pressure did not change during the period when the data were obtained (Fig. 5). In some of the 11-day embryos, data were first obtained at $10 \mathrm{~min}$ after excision.

In a summary of the data taken at the start of recording the pressure in each embryo, the heart rate was $88 \pm 11$ in the 11 day group, $122 \pm 3$ in the 12-day group, $192 \pm 7$ in the 13-day group, and $198 \pm 5$ in the 15-day group. Mean blood pressure was $0.27 \pm 0.05 \mathrm{~mm} \mathrm{Hg}, 0.48 \pm 0.03 \mathrm{~mm} \mathrm{Hg}, 1.3 \pm 0.08 \mathrm{~mm}$ $\mathrm{Hg}$, and $2.6 \pm 0.1 \mathrm{~mm} \mathrm{Hg}$, respectively (Figs. 6 and 7). Systolic and diastolic pressures increased similarly (Table 2), and pulse pressure (difference between systolic and diastolic pressures) also increased as the gestational days increased.

With respect to the embryo wet weight, the mean blood pressure of the rat embryo was compared with that of the chick embryos (Fig. 8). The data of the chick embryo were adopted from Clark and $\mathrm{Hu}(18)$. The pressure was higher in the chick embryo at stages 18 and 21 than in the rat embryo of gestational days 11 and 12, and this relationship was inverted later.

Effect of caffeine infusion. In the chick embryo, heart rate was not changed by caffeine infusion, but blood pressure was increased from $0.98 \pm 0.05$ to $1.08 \pm 0.05 \mathrm{~mm} \mathrm{Hg}(111 \pm 3 \%) 1$ $\min$ after infusion $(n=8)$. The dorsal aortic flow increased from $0.65 \pm 0.04$ to $0.69 \pm 0.06 \mathrm{~mm}^{3} / \mathrm{s}(106 \pm 6 \%, n=9)$, but the change was not statistically significant.

In the rat embryo, caffeine increased the heart rate from 136 \pm 3 to $148 \pm 4(108 \pm 2 \%) 1$ min after infusion $(n=10)$, and the mean blood flow velocity at the truncus by $21 \pm 8 \%(n=8)$. Blood pressure was changed from $0.65 \pm 0.05$ to $0.67 \pm 0.04$ $\mathrm{mm} \mathrm{Hg}(103 \pm 3 \%, n=10)$ which was not statistically significant (Fig. 9). Volume infusion itself did not affect these parameters in either chick or rat embryos (Fig. 9).

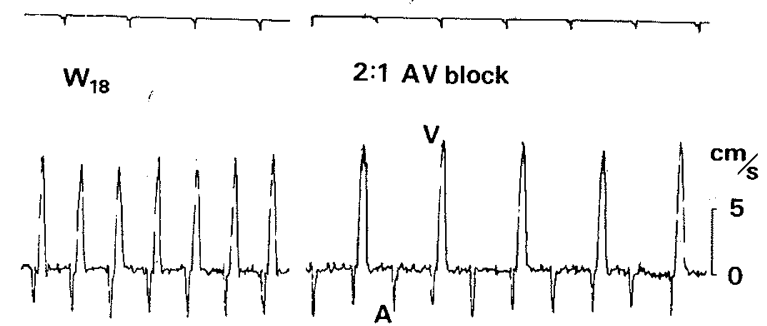

Fig. 4. When the Doppler beam was directed caudally, a negative signal was recorded. In the case with 2 to 1 atrioventricular block, the negative signal $(A)$ was found to result from atrial systolic output into the primitive ventricle. $V$, ventricular output.

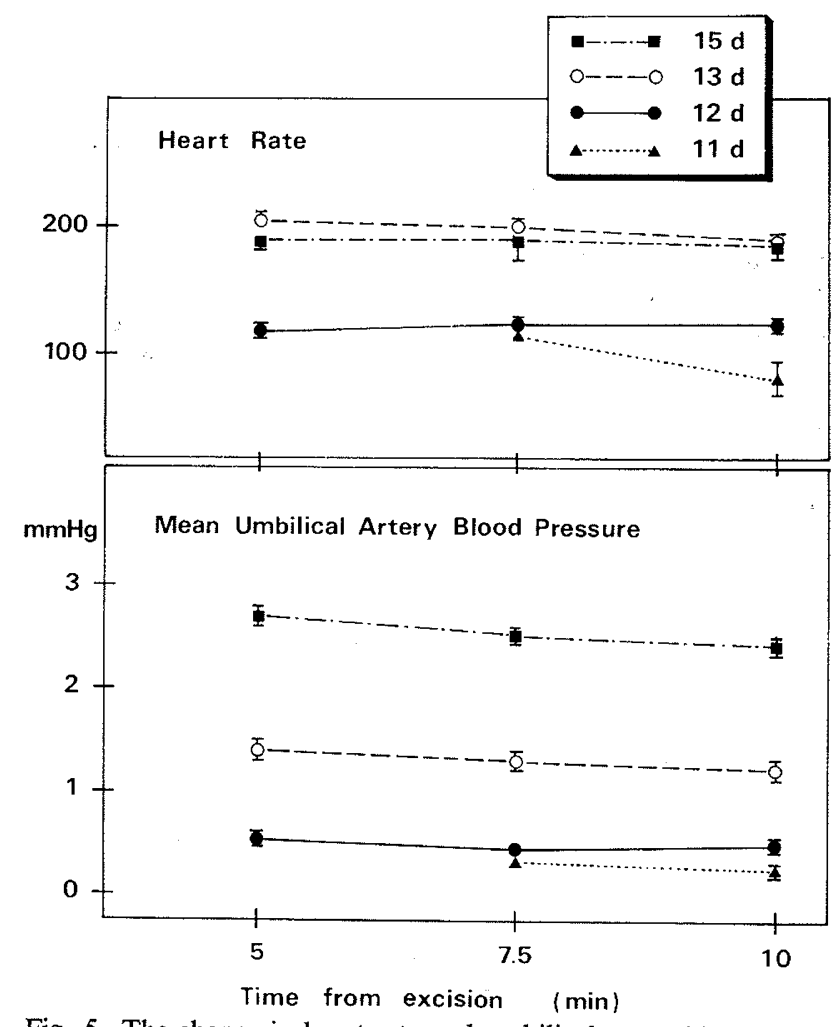

Fig. 5. The change in heart rate and umbilical artery blood pressure was plotted against the time from excision of the embryo from the mother rat. Both parameters are very stable in the group of gestational day 12 . In the group of day 11 embryos, the number of data was not large enough to reach a conclusion (see the text). In the older groups, these parameters tended to decrease slightly with time but the changes were not statistically significant.

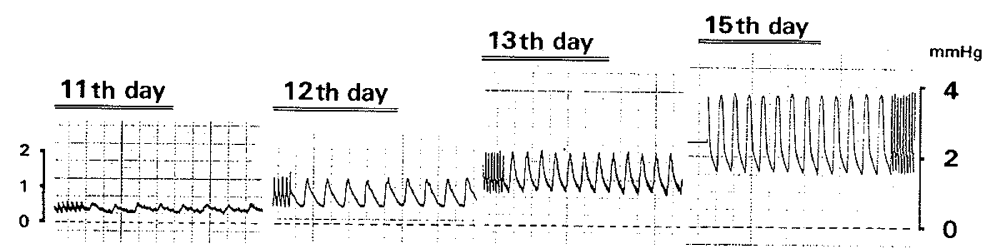

Fig. 6. Actual tracings of umbilical artery blood pressure at each gestational day. 


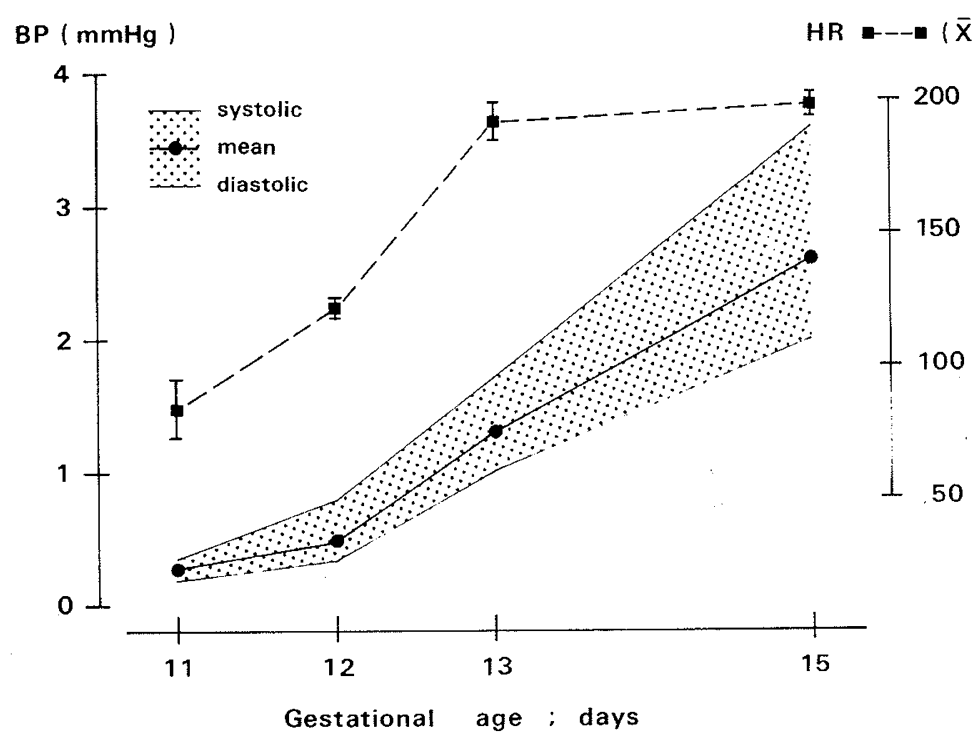

Fig. 7. Changes in the blood pressure and heart rate with development. The solid line within the hatched area indicates the average mean

blood pressure. The upper and lower border of the hatched area indicates the mean systolic and diastolic pressures, respectively.

Table 2. Developmental change of systolic and diastolic pressures [mean \pm SEM $(\mathrm{mm} \mathrm{Hg})$ ]

\begin{tabular}{lcccc}
\hline Gestational day & 11 & 12 & 13 & 15 \\
$n$ & 7 & 19 & 11 & 10 \\
\hline Systolic pressure & $0.34 \pm 0.07$ & $0.79 \pm 0.05$ & $1.7 \pm 0.11$ & $3.6 \pm 0.1$ \\
Diastolic pressure & $0.19 \pm 0.03$ & $0.34 \pm 0.02$ & $1.0 \pm 0.08$ & $2.0 \pm 0.1$ \\
\hline
\end{tabular}

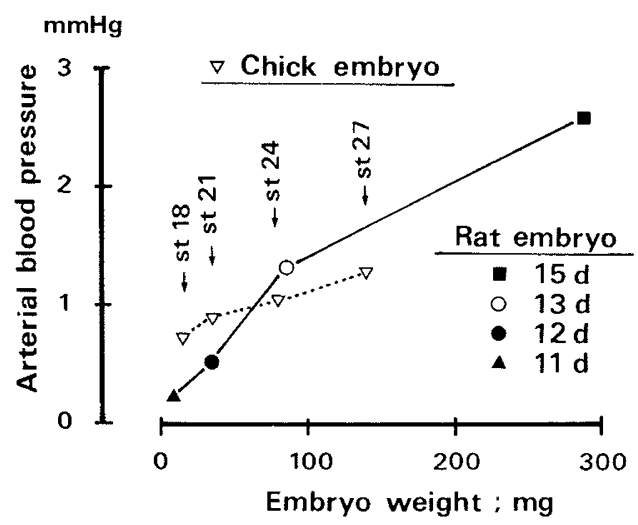

Fig. 8. Comparison of the change in blood pressure in the rat and chick embryos with respect to wet weight of the embryo. The data of the chick embryo was adopted from Clark and $\mathrm{Hu}(18)$. The pressure was lower in the rat embryo than in the chick embryo during the early stage.

\section{DISCUSSION}

The most critical problem for our preparation was the fact that the embryonic circulation was connected with the allantoic placenta and with the yolk sac circulation, to which the blood supply was interrupted. New $(21,22)$ applied an organ culture technique to the rat embryo. The embryo was excised from the pregnant rat at various gestational days and put it into a fully oxygenated culture medium containing rat serum. The embryo explanted at 8.5 or 9.5 days of gestation could be grown for 2-3 days.

The yolk sac is the site where the embryo extracts oxygen from the perfusate before the allantoic placenta starts to function, so that the young embryos are able to survive in the culture medium without normally functioning allantoic placenta $(21,22)$. New $(21,22)$ has shown that opening of the yolk sac is advantageous for the culture even in the 11-day embryo or later when the
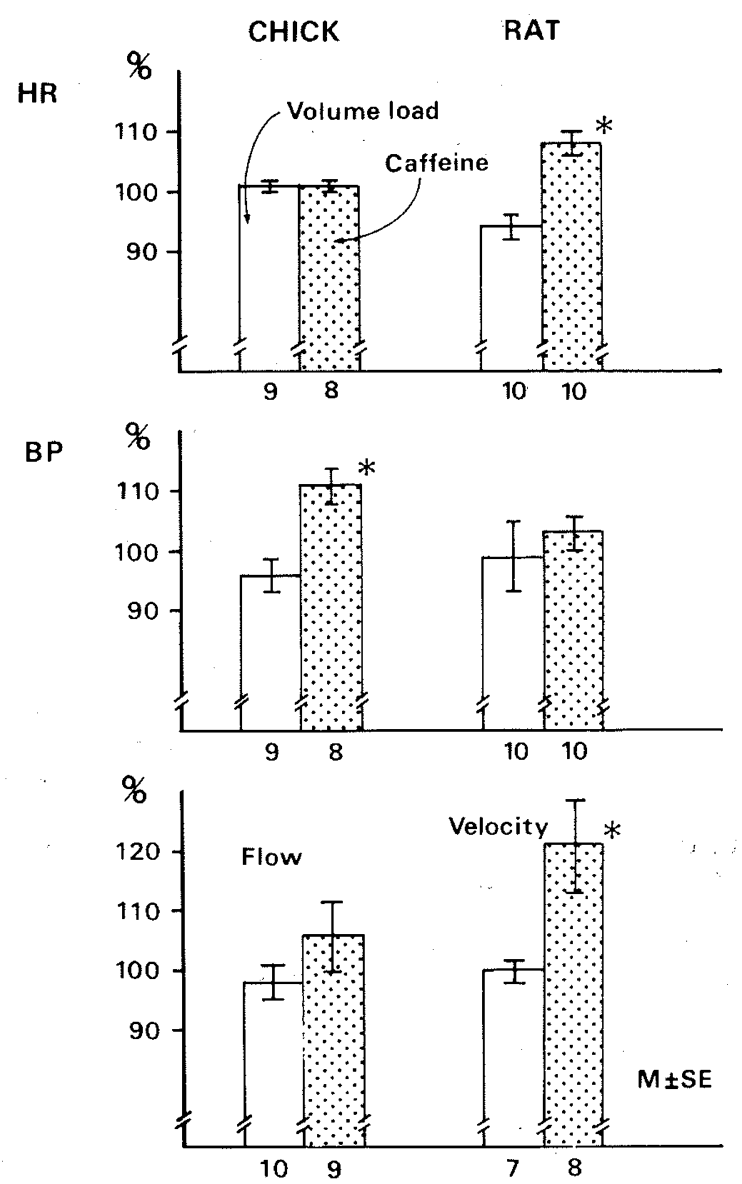

Fig. 9. The effect of caffeine on the hemodynamic parameters are presented as percent change from the baseline on the left for the rat embryo and on the right for the chick embryo. Open bars indicate the effect of volume infusion itself on the same parameters for both embryos. The numbers on the abscissa for each bar are the $n$. Caffeine induced an elevation of blood pressure $(B P)$ and a modest, not statistically significant, increase of dorsal aortic flow (flow) without changing heart rate $(H R)$ in the chick embryo. In the rat embryo, it increased heart rate but did not affect blood pressure. The blood flow velocity at the conotruncus (velocity) was increased indicating cardiac output was increased by caffeine. The asterisk indicates that the change is statistically significant $(p<0.05)$ from the baseline. 
allantoic placental circulation becomes predominant over the yolk sac circulation. The opening should increase the surface area of the yolk sac in contact with the perfusate; thus, extraction of oxygen would become more effective.

These findings indicate that the rat embryo can survive for a limited period out of the uterus under certain conditions and that younger embryos are apt to survive for a longer period in culture medium than older embryos. The data obtained in the present study are in agreement with these observations. The embryo was in a stable condition for the first $10 \mathrm{~min}$ in the Hanks' solution, especially the 12-day embryo. The older embryos also showed relatively stable data during the same period, although modest declines in heart rate and blood pressure were observed. The latter might be an expression of hypoxemia of the embryo because older embryos must be more susceptible in a depletion of oxygen because the role of the yolk sac circulation becomes less prominent than that of the allantoic placental circulation. This finding indicates that this preparation is useful as an acute experimental model especially in the day-12 embryo, but hypoxia could limit the usefulness of this preparation for more lengthy studies.

Also, it is questionable whether the umbilical artery blood pressure is completely equal to the embryonic blood pressure because the younger embryo is at the stage of transition of the circulation from the yolk sac to the placenta, thus the umbilical vessels may not be fully patent or may be stenotic. Generally, any pressure loss (pressure gradient) through the flow is a result of energy loss due to turbulence distal to a localized stenosis. Although a precise study has not been undertaken, we have speculated that the umbilical artery becomes physiologically patent from the fetal side because no potential exists at the placental side but only in the blood pressure and flow of the fetus. In this situation, it is not probable that there is any localized stenosis throughout the umbilical artery proximal to the site where the pressure was measured. A transient vasoconstriction may occur, however, that should have a larger hemodynamic effect in the smaller vessel of the younger embryo, which may explain the relatively low pressure and narrow pulse pressure seen in the younger embryos. However, if this had actually happened in the present subject the pressure should have fluctuated during recordings and we did not observe this in any embryo.

Robkin et al. (23) investigated the effect of catecholamines on the heart rate in the rat embryo using an organ culture technique of New $(21,22)$. They used the laser fluorometric method to count heart rate and showed that it was more than 150 beats/ min before the intervention on the 11th day of gestation. This number is obviously different from our current data. They used a temperature of $38.5^{\circ} \mathrm{C}$ whereas it was $37^{\circ} \mathrm{C}$ herein, which could be one of the factors of the difference. Robkin et al. (24) demonstrated that a change in temperature of $1^{\circ} \mathrm{C}$ altered the heart rate by $7 \%$. Taking this figure into account, the heart rate herein was still somewhat lower than theirs. As suggested previously, this might have resulted from the characteristics of our preparation.

Inasmuch as it is currently not possible to measure these parameters in the in situ embryo, the only way to estimate them is the in vitro study, in which the physiological environment is made as close to the in vivo condition as possible. In this aspect, the preparation presented herein is not yet complete, and we do not know how the procedure has affected the interrelationship between allantoic placenta and yolk sac contributions to fetal blood supply, thus, the hemodynamic data. However, the stability of the parameters within 10 min after excision (Fig. 6) indicates that the present data would represent the in vivo condition with a fairly good accuracy, at least for acute measurements.

Herein we demonstrated that the increase in blood pressure was parallel with the increase in embryo body weight. Also observed was the widening of pulse pressure that should have resulted from the increase in stroke volume. This, along with the increase in heart rate, indicates that cardiac output should also increase very rapidly to meet the increase of metabolic demand of the rapidly growing embryo during this period of development.

The basic differences between the hemodynamic condition of the chick and rat embryos is in the system supporting the embryonic circulation; the rat embryo is connected to the placenta whereas the chick embryo is supported by the vitelline circulation. Thus, it might be speculated that this difference caused the difference in blood pressure during the early stage of development (Fig. 9).

The administration of caffeine also resulted in different responses in these species. Hawkins et al. (8) demonstrated that caffeine, at a dose of $1.2 \times 10^{-2} \mathrm{mg}$, increased vitelline arterial blood pressure and cardiac output in the chick embryo. Herein the change in blood pressure was the same as in their study (8) but the increase in the dorsal aortic flow was not statistically significant, and this may have resulted from the smaller dose of the drug $\left(2 \times 10^{-3} \mathrm{mg}\right)$ used in our study.

The elevation of blood pressure could have resulted either from the increase of flow within a high-resistant artery system, or from the active vasoconstriction along with an increase in output both induced by the drug. In either case, the presence of a resistant vitelline circulation system played an essential role to the hemodynamic characteristics observed in the chick embryo.

In the rat embryo, there is no way to estimate cardiac output at present. As demonstrated herein, blood flow velocity was obtained at the truncus. However, the instantaneous measurement of the truncus diameter is not possible in the in vivo embryonic heart. We tried to measure the internal diameter of the truncus in the formalin-fixed embryo. We found that the conotruncal swelling already had valve function at these stages, which was also presented by the velocity tracing in Figure 3 , and that the position of the "valve" was not constant among the materials. In addition, the diameter of the truncus distal to those swellings also varied largely among embryos with similar body size. Thus, it was not possible to determine the diameter with any standard, so we could not obtain the absolute value of cardiac output by this approach. If one could obtain flow velocity at any large vessel such as the dorsal aorta, the absolute value of output could have been calculated simply by measuring the diameter of the vessel. However, none of large vessels was clearly seen through the embryo body.

Assuming that the change in cardiac output was parallel to the change in velocity of the blood flow at the outflow tract in the embryonic heart, the present data indicate that caffeine increased cardiac output also in the rat embryo. The increase of cardiac output did not bring about a significantly large elevation of blood pressure. This indicates that the resistant vessels were dilated or a large reservoir was present. The latter would be the case because the intravascular capacity seems to be much larger in the placenta than in the vascular bed of the embryo at this gestational age and the placenta is a hemodynamically low resistant organ with, presumably, a large capacity.

Thus, herein we have clarified that the hemodynamic effects of caffeine were different in these two species. This difference will be related to their supporting circulation systems (vitelline versus placental circulations), or may possibly be related to differences in the development of the physiological functions of the cell, including receptors.

Fujii and Nishimura (14) and Matsuoka et al. (15) demonstrated that caffeine given to pregnant rats caused ventricular septal defect in their embryos. These morphological studies and our present findings support the view of Clark (5) who suggested that an increase in left heart blood flow during ventricular septation may interfere with the normal morphogenesis of the septum, thus resulting in ventricular septal defect. In contrast, the same agent caused aortic aneurysm in the chick embryo (13). In general, the elevation of blood pressure should cause an increase of aortic wall tension. If this happens during morpho- 
genesis, it is possible that the hemodynamic change results in the abnormal morphology. This might explain, at least in part, the genesis of aortic aneurysm in the chick embryo.

It is obvious that these acute experimental data cannot be extrapolated directly to chronic experimental or clinical data. The major objections would be the dosage of teratogenic agent, duration of exposure to the agent, and placental transfer when the agent was given to a pregnant rat. Inasmuch as the concentration of caffeine in the embryo was not given in the morphological studies cited above, we could not determine how the dose used in the herein compared to that used in these studies. It is known that caffeine readily crosses the placenta in humans (25) and in the rat and in the fetus the drug was recovered to as much as $50 \%$ of that in the mother, when normalized by body weight (26). Thus, the dosage of our study would not be very much different to that of these morphological studies, if it was expressed as $\mathrm{mg} / \mathrm{kg}$ of body weight.

It is questioned if the duration of exposure was long enough to exert teratogenecity. Rychter (27) suggested that an exposure to teratogene even for a very short period could possibly be a cause of abnormal morphogenesis. Indeed, Bruyere et al. (28) reported an interesting observation when they gave epinephrine to the chick embryo at stage 19 to 21 while recording the change of heart size under a micro-cinecamera. An embryo, which showed an increase of cardiac output 1 min after the intervention, later developed an overriding aorta and ventricular septal defect. In contrast, another embryo, in which cardiac output decreased for the same period, later developed a hypoplastic aortic arch. Thus, it is possible to speculate that the difference of acute hemodynamic response to caffeine seen herein is related, at least in part, to the difference in morphogenesis between the chick and rat embryos.

The present study indicates that measurement of blood pressure in the rat embryo is feasible, but with some limitations, and that there may be qualitative hemodynamic differences between the rat and chick embryos.

\section{REFERENCES}

1. Jaffee OC 1965 Hemodynamic factors in the development of the chick embryo heart. Anat Rec 151:69-76

2. Jaffee OC 1966 Rheological aspects of the development of blood flow patterns in the chick embryo heart. Biorheology 3:59-62

3. Jaffee OC 1967 The development of the arterial outflow tract in the chick embryo heart. Anat Rec 158:35-42

4. Gilbert EF, Bruyere HJ, Ishikawa S, Foulke LM, Heimann SR 1980 Role of decreased cardiac output in isoproterenol-induced cardiovascular teratogenesis in chick embryo. Teratology 21:299-307

5. Clark EB 1987 Mechanism in the pathogenesis of congenital cardiac malformations. In: Pierpont MEM, Moller JH (eds) Genetics of Cardiovascular Disease. Martinus Ninhoff Publ, Boston, pp 3-11
6. Yoshida H, Manasek F, Arcilla RA 1980 Intracardiac flow patterns in early embryonic life: reevaluation. Circ Res 53:363-371

7. Clark EB, Hu N, Dooley JB 1985 The effect of isoproterenol on cardiovascular function in the stage 24 chick embryo. Teratology 31:41-47

8. Hawkins JA, Hu N, Clark EB 1984 Effect of caffeine on cardiovascular function in the stage 24 chick embryo. Dev Pharmacol Ther 7:334-343

9. Wispe J, Hu N, Clark EB 1983 Effect of environmental hypothermia on dorsa aortic blood flow in the chick embryo, stage 18 to 24 . Pediatr Res 17:945948

10. Nakazawa M, Clark EB, Hu N, Wispe J 1985 Effect of environmental hypothermia on vitelline artery blood pressure and vascular resistance in the stage 18, 21, and 24 chick embryo. Pediatr Res 19:651-654

11. Nakazawa M, Miyagawa S, Takao A, Clark EB, Hu N 1986 Hemodynamic effects of environmental hyperthermia in stage 18,21 , and 24 chick embryos. Pediatr Res 20:1213-1215

12. Stewart DE, Kirby ML, Sulik KK 1986 Hemodynamic changes in chick embryos precede heart defects after cardiac neural crest ablation. Circ Res 59:545-550

13. Gilbert EF, Bruyere HJ, Ishikawa S, Cheung MO, Hodach RJ 1977 The effects of methylxanthines on catecholamine-stimulated and normal chick embryos. Teratology 16:47-52

14. Fujii T, Nishimura H 1972 Adverse effects of prolonged administration of caffeine on rat fetus. Toxicol Appl Pharmacol 22:449-457

15. Matsuoka R, Uno H, Gilbert EF, Tanaka H, Kerr CS, Nadal-Ginard B 1987 Caffeine induces cardiac and other malformations in the rat. Am $\mathrm{J}$ Med Genet (in press)

16. Sissman NJ 1978 Developmental landmarks in cardiac morphogenesis: comparative chronology. Am J Cardiol 25:81-122

17. Hamburger V, Hamilton HL 1951 A series of normal stages in the developmen of the chick embryo. J Morphol 88:49-92

18. Clark EB, Hu N 1982 Developmental hemodynamic changes in the chick embryo from stage 18 to 27 . Circ Res 51:810-815

19. Nakazawa M, Miyagawa S, Atsuyoshi Takao 1986 Developmental hemody namic changes in the rat embryo from 11 to 15 gestational days. Circulation 74(suppl-II):II-295(abstr)

20. Beaudoin AR 1980 Embryology and teratology. In: Baker HJ, Lindsey R, Weisbroth SH (eds) The Laboratory Rat, Vol 2. American College Laboratory. Academic Press, New York, pp 75-101

21. New DAT 1975 Studies on mammalian embryos in vitro during the period of organogenesis. In: Shepard TH, Miller JR, Marois M (eds) Methods for Detection of Environmental Agents that Produce Congenital Defects. NorthHolland Publ Co, Amsterdam, pp 145-160

22. New DAT 1978 Whole-embryo culture and the study of mammalian embryos during organogenesis. Biol Rev 53:81-122

23. Robkin M, Shepard TM, Baum D 1974 Autonomic drug effect on the heart rate of early rat embryos. Teratology 9:35-44

24. Robkin MA, Shepard TM, Tanimura T $1972 \mathrm{~A}$ new in vitro culture technique for rat embryos. Teratology 5:367-376

25. Goldstein A, Warren R 1962 Passage of caffeine into human gonadal and fetal tissue. Biochem Pharmacol 11:166-168

26. Arnaud MJ, Bracco I, Sauvageat JL, Clerc M-F 1983 Placental transfer of the major caffeine metabolite in the rat using 6-amino-5 [N-formylmethylamino] 1,3 [Me-14C]-dimethyluracil administered orally or intravenously to the pregnant rat. Toxicol Lett 16:271-279

27. Rychter Z 1962 Experimental morphology of the aortic arches and heart loop in chick embryos. Adv Morphol 2:333-371

28. Bruyere HJ Jr, Folts JD, Gilbert EF 1984 Hemodynamic mechanisms in the pathogenesis of cardiovascular malformations in the chick embryo: cardiac function changes following epinephrine stimulation in chick embryos. In: Nora JJ, Takao A (eds) Congenital Heart Disease: Cause and Processes. Futura, New York, pp 279-292 